García-Holgado, A., \& García-Peñalvo, F. J. (2018). Mapping the systematic literature studies about software ecosystems. In F. J. García-Peñalvo (Ed.),

Proceedings TEEM'18. Sixth International Conference on Technological Ecosystems for Enhancing Multiculturality (Salamanca, Spain, October 24th-26th, 2018)

(pp. 910-918). New York, NY, USA: ACM. doi: 10.1145/3284179.3284330

\title{
Mapping the systematic literature studies about software ecosystems
}

\author{
Alicia García-Holgado \\ GRIAL Research Group, \\ Research Institute for Educational Sciences, University of \\ Salamanca \\ 37008 Salamanca, Spain \\ aliciagh@usal.es
}

\author{
Francisco José García-Peñalvo \\ GRIAL Research Group, \\ Research Institute for Educational Sciences, University of \\ Salamanca \\ 37008 Salamanca, Spain \\ fgarcia@usal.es
}

\begin{abstract}
There is a need to improve the definition and development of technological ecosystems in order to solve the main problems detected in previous studies. To achieve this goal, it is required to identify and analyse the solutions available in the literature in the field of software engineering applied to ecosystems. The research in software ecosystems is a relatively young research area, but there are already several works that analyse the literature associated. To conduct a new systematic literature review is necessary to ensure that there are no studies that do the same, namely, that do not answer the same research questions. The identification of the need for a review was done through a study focused on systematic literature reviews and mapping studies about software ecosystems. This work aims to describe the mapping conducted as part of that study. It provides a global state of the art of this kind of studies in the area of software ecosystems.
\end{abstract}

\section{CCS CONCEPTS}

- Information systems • Software and its engineering $\rightarrow$ Software creation and management $\cdot$ Software and its engineering $\rightarrow$ Ultra-large-scale systems

\section{KEYWORDS}

Technological ecosystems, software ecosystems, software engineering, systematic mapping, systematic literature review.

\section{INTRODUCTION}

The metaphor of ecosystem, which comes from the area of biology, is used in different contexts to convey the evolutionary nature of processes, activities, and relationships. From a technological point of view, Iansiti and Levien [1] are the first to relate technology to business ecosystems by claiming that they evolve around a platform. According to Manikas [2] the research in software ecosystems (SECOs) starting in 2005 with the book by Messerschmitt and Szyperski [3].

There are different definitions of SECO in the literature [4-8]. Moreover, although software ecosystem is the most common term, there are some authors that use other terms such as digital ecosystem [9,10], service ecosystem [11] or technological ecosystem [12, 13], among others, to talk about a set of software components that are related to each other in some way to give rise to more complex systems. Each term has different shades. Specifically, the authors of this work use the term "technological ecosystem" to highlight the evolutionary characteristics and the human factor as part of the ecosystem, which is composed by heterogeneous software components without a central platform or element.

There is a need to improve the definition and development of technological ecosystems in order to solve the main problems detected in previous studies $[12,14]$. To reach this goal, it is required to identify and analyse the solutions available in the literature in the field of software engineering applied to ecosystems.

The systematic literature review (SLR), a methodology proposed by [15], provides the guidelines to identify and analyse the literature associated with a specific topic. The first activity defined by this methodology is the identification of the need for a review. Before planning and conducting an SLR about solutions of software engineering for defining and developing software ecosystems, it is required to identify if there are other SLR in the literature with the same objective. To do this, an SLR focused on systematic literature reviews and mapping studies about software ecosystems was conducted.

This work aims to describe the mapping conducted to complement the systematic literature review about SLR of software ecosystems. 
The paper is divided into five sections. The first part describes the planning phase of the mapping study. The second part presents the data extraction process. The third part answers the mapping questions. The fourth part analyses the results. Finally, the last part summarizes the main conclusions of this work.

\section{RESEARCH METHOD}

This research is based on the guidelines provided by Kitchenham and Charters [15] for systematic literature reviews and the guidelines provided by Petersen [16, 17] for mapping studies. In particular, the process is organized in three main phases: planning, conducting and reporting the study.

\subsection{Research questions}

The overall research objective of this study is to find and analyse the systematic literature reviews and mappings about software ecosystems. In particular, the systematic mapping aims to answer the following questions:

- MQ1. How many studies were published over the years?

- MQ2. Who are the most active authors in the area?

- MQ3 What type of papers are published?

- MQ4. In which sources appear this kind of studies?

- MQ5. Which are the most common databases used in this kind of studies?

- MQ6. Which search terms are used to define the search string in this kind of studies?

- MQ7. In which domains are the studies focused?

- MQ8. Which years cover the review and mapping studies?

- MQ9. What type of review are published?

Then, the PICOC method proposed by Petticrew and Roberts [18] was used to define the scope of the review:

- $\quad$ Population (P): systematic literature reviews and mappings.

- Intervention (I): conduct a systematic literature review about software architecture and model driven engineering in technological ecosystems.

- Comparison (C): no comparison.

- $\quad$ Outputs $(\mathrm{O})$ : the systematic reviews and mappings about technological ecosystems.

- $\quad$ Context $(\mathrm{C})$ : contexts related to technological and software ecosystems.

\subsection{Inclusion and exclusion criteria}

A set of inclusion and exclusion criteria was defined to select those works that are relevant to answer the defined research questions. Specifically, five inclusion criteria (CI) and the corresponding five exclusion criteria (EC) was defined:

- IC1: The paper describes a literature review or mapping focused on software or technological ecosystems AND

- IC2: The presented literature review or mapping follows a systematic process AND

- IC3: The paper is written in English AND

- IC4: The paper is published in peer review journals, books, conferences or workshops AND

- IC5: The full version of the paper is available through the authors' institution or through an authors' membership to an association. Regarding the exclusion criteria:

- $\quad$ EC1: The paper does not describe a literature review or mapping focused on software or technological ecosystems OR

- $\quad$ EC2: The presented literature review or mapping does not follow a systematic process OR

- $\quad$ EC3: The paper is not written in English OR

- $\quad$ EC4: The paper is not published in peer review journals, books, conferences or workshops OR

- $\quad$ EC5: The full version of the paper is not available through the authors' institution or an authors' membership to an association.

\subsection{Search strategy}

First, the selected sources to search are four electronic databases: Scopus, Web of Science (WoS), IEEE Xplorer and ACM Digital Library. These databases were chosen according to a set of requirements:

- It is a reference database in the research scope.

- It is a relevant database in the research area in this mapping study.

- It allows using a search string equal or similar to the rest of the selected databases.

- It is a database available through the authors' institution or an authors' membership to an association. 
Regarding the search terms, they were identified from the PICOC and the synonymous or alternatives used in the literature to talk about systematic reviews and technological ecosystems.

In particular, the chosen search terms related to ecosystems are: technological ecosystem, software ecosystem, SECO, information ecosystem, ERP ecosystem, open ecosystem, learning ecosystem.

On the other hand, the terms related to systematic literature reviews are: SLR, Systematic Literature Review, systematic mapping, literature review.

\subsection{Search strings}

The search strings for each chosen source were defined from the search terms connected by boolean AND / OR operators. Moreover, the wildcard $\left(^{*}\right)$ was used in Scopus and WoS to include both singular and plural of each term.

No restrictions about time were applied in the search. Namely, the results were not limited by publication year of the papers. Regarding the thematic areas or categories, some restrictions were applied in WoS and Scopus to avoid papers related to biological ecosystems. In particular, the "VETERINARY SCIENCES" and “AGRICULTURE DAIRY ANIMAL SCIENCE" categories were excluded in WoS and the "Agricultural and Biological Sciences" and "Social Sciences" thematic areas in Scopus. Regarding the document types, only papers in conferences, articles in journals, book chapters and reviews were included.

The chosen terms were searched in the title, the keywords and the abstract of the papers. The base query was the following:

("technological ecosystem*" OR "software ecosystem*” OR SECO OR "information ecosystem*” OR "ERP ecosystem" OR "open ecosystem*” or "learning ecosystem*”) AND (SLR OR "Systematic Literature Review" OR "systematic mapping” OR "literature review")

The search strings per each chosen electronic database are as follow:

WoS:

$$
\begin{aligned}
& T S=((\text { "technological ecosystem *” OR "software ecosystem *” OR SECO OR "information ecosystem *” OR "ERP ecosystem" "OR "open } \\
& \text { ecosystem *” or "learning ecosystem*”) AND (SLR OR “Systematic Literature Review" OR “systematic mapping” OR "literature review”)) }
\end{aligned}
$$

- Scopus:

TITLE-ABS-KEY ( "technological ecosystem*” OR "software ecosystem*” OR seco OR "information ecosystem" OR "ERP ecosystem *” OR "open ecosystem*" OR "learning ecosystem*") AND TITLE-ABS-KEY ( slr OR "Systematic Literature Review" OR "systematic mapping" OR "literature review”) AND ( LIMIT-TO ( DOCTYPE, "cp”) OR LIMIT-TO ( DOCTYPE, “ar") OR LIMIT-TO ( DOCTYPE, "ch") OR LIMIT-TO (DOCTYPE, "re”)) AND (EXCLUDE ( SUBJAREA, “AGRI”) OR EXCLUDE ( SUBJAREA, "MEDI”) OR EXCLUDE ( SUBJAREA, "SOCI")) AND (LIMIT-TO (LANGUAGE, "English"))

- IEEE Xplorer:

("technological ecosystem" OR "software ecosystem" OR SECO OR "information ecosystem" OR "ERP ecosystem" OR "open ecosystem" or "learning ecosystem”) AND (SLR QR "Systematic Literature Review” OR "systematic mapping” OR "literature review”)

- $\quad$ ACM Digital Library:

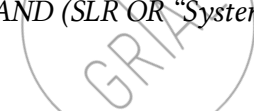

((acmdlTitle: ("technological ecosystem" OR "software ecosystem" OR SECO OR "information ecosystem" OR "ERP ecosystem" OR "open ecosystem" OR "learning ecosystem") OR recordAbstract: ("technological ecosystem" OR "software ecosystem" OR SECO OR "information ecosystem” OR “ERP ecosystem” OR “open ecosystem” OR “learning ecosystem”)) AND (acmdlTitle: (SLR OR "Systematic Literature Review”

OR "systematic mapping" OR "literature review") OR recordAbstract: (SLR OR "Systematic Literature Review" OR "systematic mapping” OR

"literature review")))

\section{DATA EXTRACTION}

The data extraction process is an iterative and incremental process that was divided into several stages in which different activities are carried out. To describe the process, a PRISMA flow [19] (Figure 1) is used.

First, the results obtained after applying the search strings were identified. For this, the results were downloaded in CSV (commaseparated values) format, stored in a repository in GitHub [20] and organized in a spreadsheet in Google Sheets (http://bit.ly/2yz5n3Y). The spreadsheet is configured to automatically detect duplicate titles in order to facilitate their search and removal. Also, each paper was marked in what sources is present. 
Secondly, the title, the abstract and the keywords of each paper were analysed, and the inclusion and exclusion criteria were applied. The papers were organized in another sheet of the spreadsheet, and each paper was marked as a candidate or not depending on the inclusion and exclusion criteria (http://bit.ly/2MJRvqu).

Finally, each candidate paper was fully read to decide if it fulfils a set of characteristics or quality criteria. During this analysis, the following questions were answered:

- Conduct a literature mapping?

- Conduct a literature review?

- Which is the domain?

- What aspects of the technological ecosystems analyse?

- Which are the research questions?

- Which years cover?

- What are the sources used?

- Which search terms are used?

After the full reading of the works, other relevant papers were identified. Concretely, one paper, which was also read in depth to answer the questions described above. All the information was organized in a third sheet of the spreadsheet (http://bit.ly/2K3D0vP). Table 1 shows the correspondence between the collected variables and the mapping questions.

\section{Table 1. Relationship between collected variables and mapping questions}

\begin{tabular}{|c|c|c|}
\hline Variable & Value & Question \\
\hline Mapping & $\begin{array}{l}\text { Logical field to indicate that } \\
\text { the study conducts a mapping }\end{array}$ & MQ9 \\
\hline Review & $\begin{array}{l}\text { Logical field to indicate that } \\
\text { the study conducts a review }\end{array}$ & MQ9 \\
\hline Domain & $\begin{array}{l}\text { Domain or domains on which } \\
\text { the study is focused }\end{array}$ & \\
\hline Period & $\begin{array}{l}\text { Range of years in which } \\
\text { chosen studies were publish }\end{array}$ & \\
\hline Databases & $\begin{array}{l}\text { Electronic databases used to } \\
\text { conduct the study }\end{array}$ & MQ5 \\
\hline Search terms & $\begin{array}{l}\text { Words used to define the } \\
\text { search query }\end{array}$ & MQ6 \\
\hline Authors & Set of names of the authors & MQ2 \\
\hline Year & Publication year & MQ1 \\
\hline $\begin{array}{c}\text { Type of } \\
\text { publication }\end{array}$ & $\begin{array}{l}\text { One of the following values: } \\
\text { article, book, chapter and } \\
\text { conference paper. }\end{array}$ & MQ3 \\
\hline Present in WoS & $\begin{array}{l}\text { Logical field to indicate if the } \\
\text { paper appears in this database }\end{array}$ & MQ4 \\
\hline Present in Scopus & Logical field to indicate if the & MQ4 \\
\hline
\end{tabular}




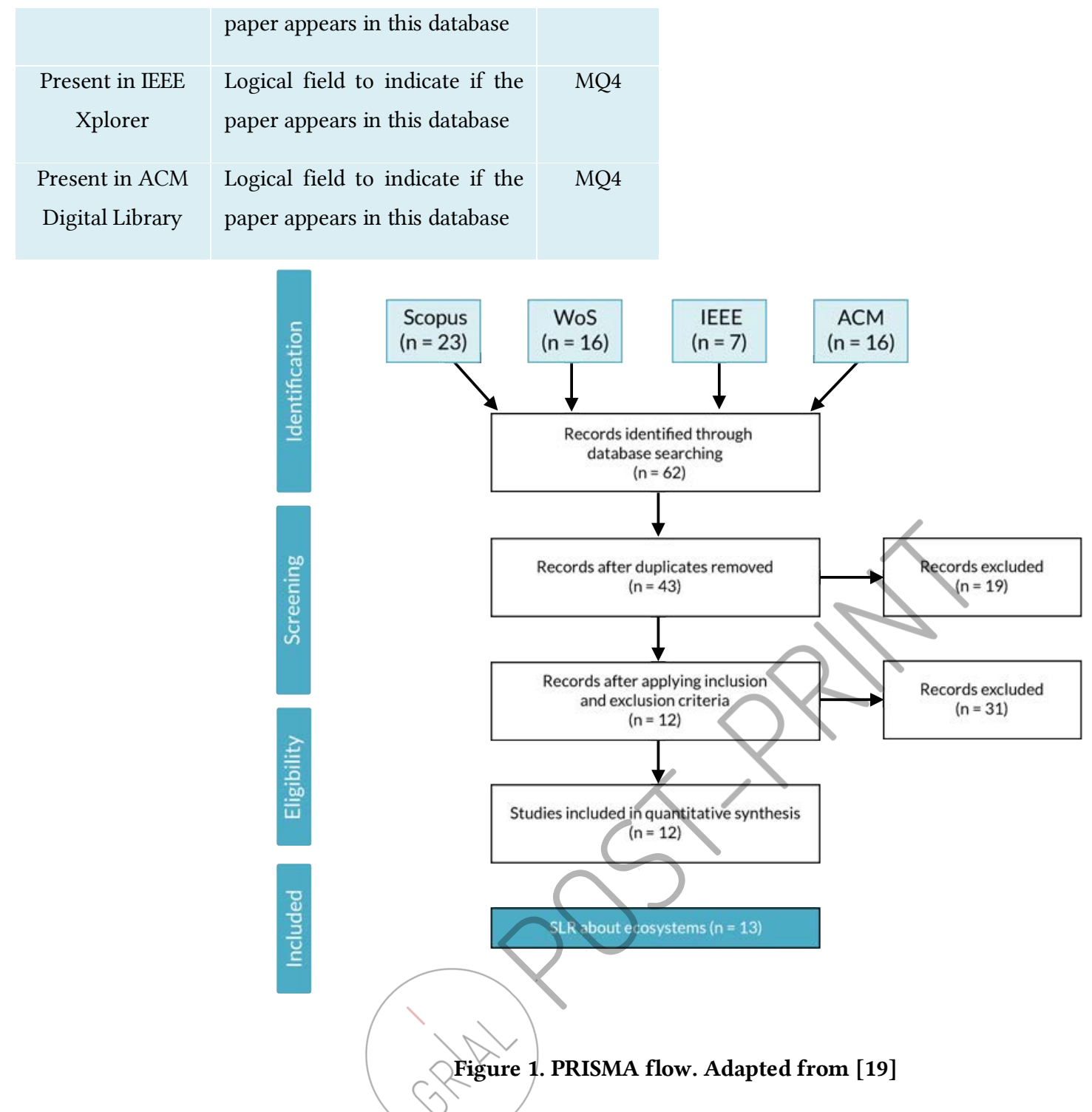

The results obtained after carrying out this process are described through a PRISMA flow [19] (Figure 1):

- After applying the search strings in each source, 62 papers were collected, of which 23 are from Scopus, 16 from WoS, 7 from IEEE Xplorer and 16 from ACM Digital Library.

- $\quad$ After removing duplicates, there are 43 papers.

- $\quad$ Once the criteria were applied to title, abstract and keywords, there are 12 papers (27.9\% of the unique papers retrieved).

- $\quad$ One paper was added after reading the selected ones.

- $\quad$ Finally, a total of 13 papers were analysed (30.23\% of the unique papers retrieved).

\section{SYSTEMATIC MAPPING RESULTS}

This section presents the data obtained after the data extraction process and the analysis of the selected papers. A Jupyter notebook (http://jupyter.org) in Python was created as technical support of this process [20]. The notebook connects to the spreadsheet in Google Sheets (http://bit.ly/2K3D0vP) and processes the data. The notebook is based on the work developed by Cruz-Benito http://bit.ly/2tS9JgF. 


\subsection{MQ1. How many studies were published over the years?}

To answer the first mapping question, the number of selected papers per year were counted. The results cover from 2011 to 2018 . The last update of the revision was made in March 2018. Figure 2 shows visually the distribution of papers per year.

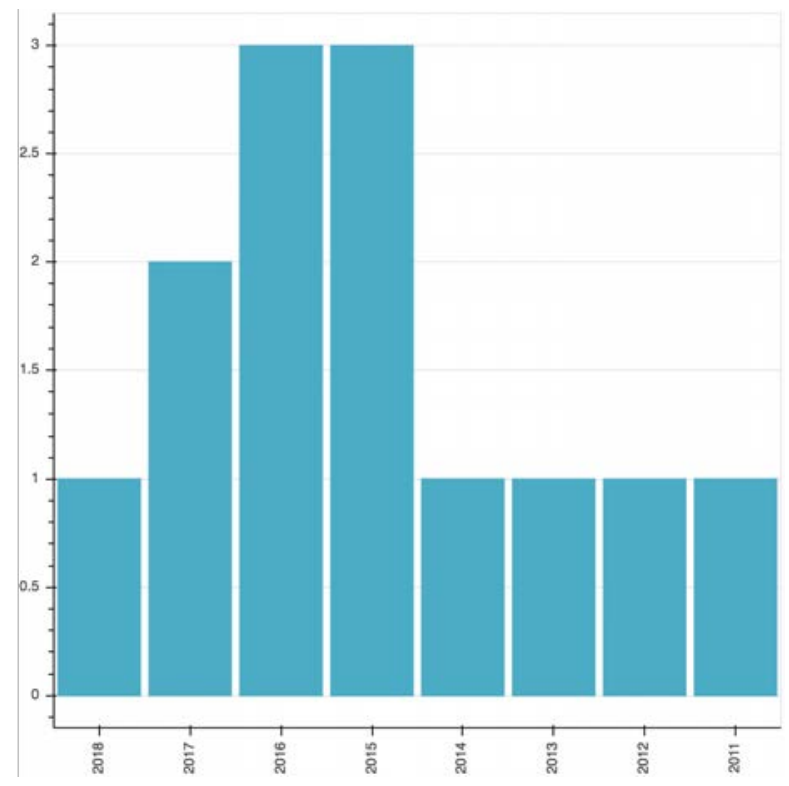

Figure 2. Graph with the number of publications per year

\subsection{MQ2. Who are the most active authors in the area?}

The second mapping question is focused on the authors of the selected works. The number of works published by each author was counted to answer this question. Although is not typical that an author publishes several systematic reviews or mappings about the same topic, some authors have conducted more than one study. In particular, Manikas appears in three works [2, 21, 22] and Alves [23, $24]$ and Andersson [25, 26] appear in two works. The other authors appear only once in the context of this mapping study. Table 2 shows the complete list of authors and the number of papers in which appear.

Table 2. Authors' name of systematic literature reviews and mapping studies about SECO

\section{Name}

\section{0 s}

Manikas, K

Alves, C.; Andersson, J

Ameller, D; Axelsson, J; Barbosa, O.; Costal, D; Dias-Neto, AC; Duc, A.N.; Dybå, T.; Fiedler, M; Fontao, AD; Fotrousi, F; Franch, 1 X; Franco-Bedoya, O; Fricker, SA; Gao, S.; Hansen, KM; Hanssen, G.; Hyrynsalmi, S; Jansen, S.; Jarvi, A; Le-Gall, F; Nokkala, T; Oliveira, J.; Papatheocharous, E; Pettersson, O; Seppanen, M; Sindre, G.; Suominen, A; Vegendla, A.; dos Santos, RP

\subsection{MQ3. What type of papers are published?}

According to the inclusion and exclusion criteria, only papers that were involved in a peer review process to be published, either in journals, conferences, books or workshops, are included.

Each electronic database provides metadata of each paper, the type of publication is part of this information. The nomenclature used was unified in order to have a clear answer to the present mapping question. In particular, the type "Proceedings Paper" used in WoS were replaced by the term used in Scopus, "Conference Paper". 
Table 3. Papers group by type of publication

\begin{tabular}{|l|l|l|}
\hline Type & Total & Papers \\
\hline Article & 4 & {$[2][22][27][28]$} \\
\hline Conference paper & 9 & {$[23][29][30][31][32][26][21][25]$} \\
& 9 & {$[24]$}
\end{tabular}

\subsection{MQ4. In which sources appear this kind of studies?}

This question allows to determine which of the four electronic databases chosen to conduct this study has the largest number of works among those selected.

Table 4 shows in which database is available the final works after conducting the search protocol. All papers are available on Scopus and most of them on WoS (9 papers). Only one final paper is available on IEEE Xplorer, and no final work is available on ACM.

Table 4. Papers group by electronic database

\begin{tabular}{|c|c|c|}
\hline Type & Total & Papers \\
\hline WoS & 9 & $\begin{array}{l}\text { [2] }[22][27][30][31][32][26][21] \\
{[25]}\end{array}$ \\
\hline Scopus & 13 & $\begin{array}{l}{[2][22][27][28][23][29][30][31]} \\
{[32][26][21][25][24]}\end{array}$ \\
\hline IEEE Xplorer & 1 & [31] \\
\hline $\begin{array}{l}\text { ACM Digital } \\
\text { Library }\end{array}$ & 0 & \\
\hline
\end{tabular}

\subsection{MQ5. Which are the most common databases used in this kind of studies?}

The first phase of a systematic literature review is focused on planning the study and defining the search protocol. The selection of the sources in which the study is conducted affects to the records collected. This mapping question aims to know which are the electronic databases chosen as sources in the reviews and mappings related to technological ecosystems (Figure 3).

The main sources are IEEE Xplorer that is used in 12 papers, followed by ACM Digital Library and ScienceDirect that appears in 11 papers. Moreover, there are two databases, Inspec and Compendex, that are available through Engineering Village which is used in 2 papers.

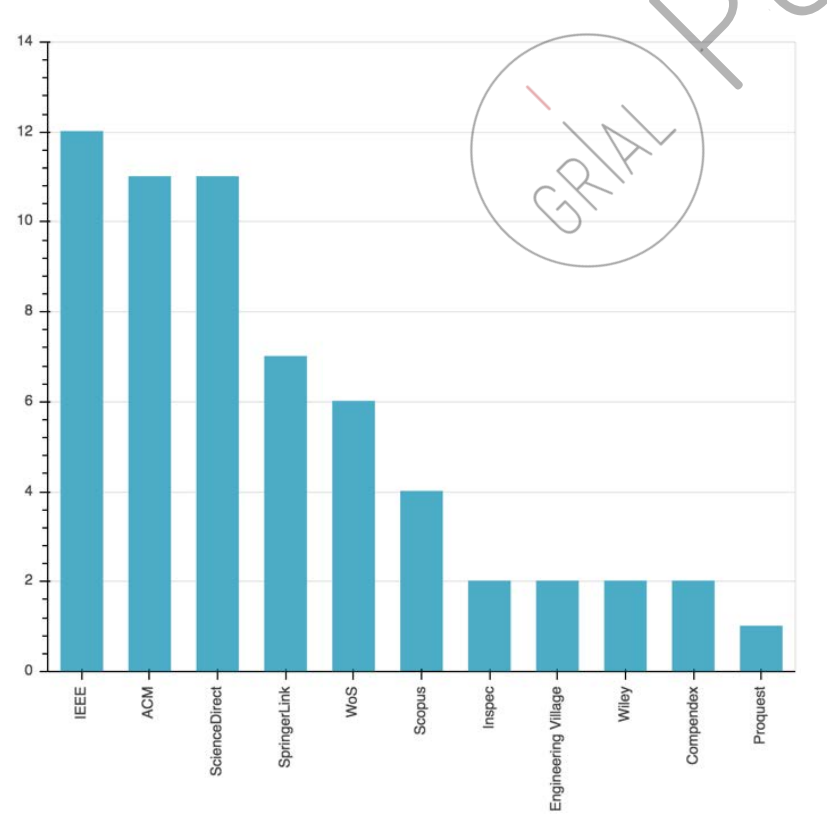

Figure 3. Graph with the number of papers that use each source 


\subsection{MQ6. Which search terms are used to define the search string in this kind of studies?}

Regarding the search terms, the most common term is "software ecosystem" which appears in 11 papers. The plural version appears in conjunction with the singular version only in 5 papers. The term "software supply network" is used by 3 papers as a way to identify software ecosystems. There are three terms that appear only in 2 papers - "business model", "ecosystem" and "significance". The other terms appear only once in the context of this mapping study. Concerning the "technological ecosystem" term is not used in any of these papers. Table 5 shows the complete list of search terms and the number of papers that use them.

Table 5. Number of papers which use each search terms

FLOSS; FOSS; ISO42010; OSS; academic community; activity; advantage; advantages; analy*; analytic; android; app; apple; application; applications; apps; area; areas; attribute; blackberry; businessmodel; challenge; challenges; characteristic; cloud ecosystem; cloud supply network; communic* ecosystem; communic ${ }^{*}$ supply network; consequence; consequences; digital ecosystem; digital supply network; discipline; disciplines; document; documentation; documenting; eco system; eco-system; elicit*; embedded; embedded software; embedded system; field; free software; game; games; google; health; ict ecosystem; ict supply network; implication; implications; indicator; industry; innovation system; ios; key characteristic; kpi; libre software; limitation; limitations; maintainability; manag*; market; marketplace; measure; metric; microsoft; mobile; mobile ecosystem; mobile supply network; model; model ${ }^{*}$; modeling; negotiate; nokia; non-functional requirement; open innovation; open source; open-source; opensource; performance; platform ecosystem; product development; product line; product-line; productline; quality attribute; reliability; requirement; restriction; restrictions; reward; safety; security; service ecosystem; service supply network; software development; software engineering; software intensive ecosystem; software intensive supply network; software quality; software supply Industry; software supply industry; software vendor; software vendors; specif*; strategic innovation; subject area; subject field; success factor; systems engineering; telecom* ecosystem; telecom* supply network testability; third party; third-party; usability; valid*; verif*; view; viewpoint; windows

\subsection{MQ7. In which domains are the studies focused?}

The systematic literature review or mappings are focused on a specific domain to analyse its state of the art, but each domain can have several subdomains or associated domains. Although all the selected studies belong to the software ecosystems domain, some studies are focused on a particular aspect or subdomain of software ecosystems (Figure 4).

Most of the papers are focused on software ecosystems in general (9). The others are focused on a particular type of software ecosystem according to two dimensions, license - open source and proprietary - and device - embedded and mobile -. In particular, regarding the license, 1 paper about open source software ecosystems and 1 paper about proprietary software ecosystems; and regarding the device, 1 paper about embedded ecosystems and 1 paper about mobile software ecosystems. 


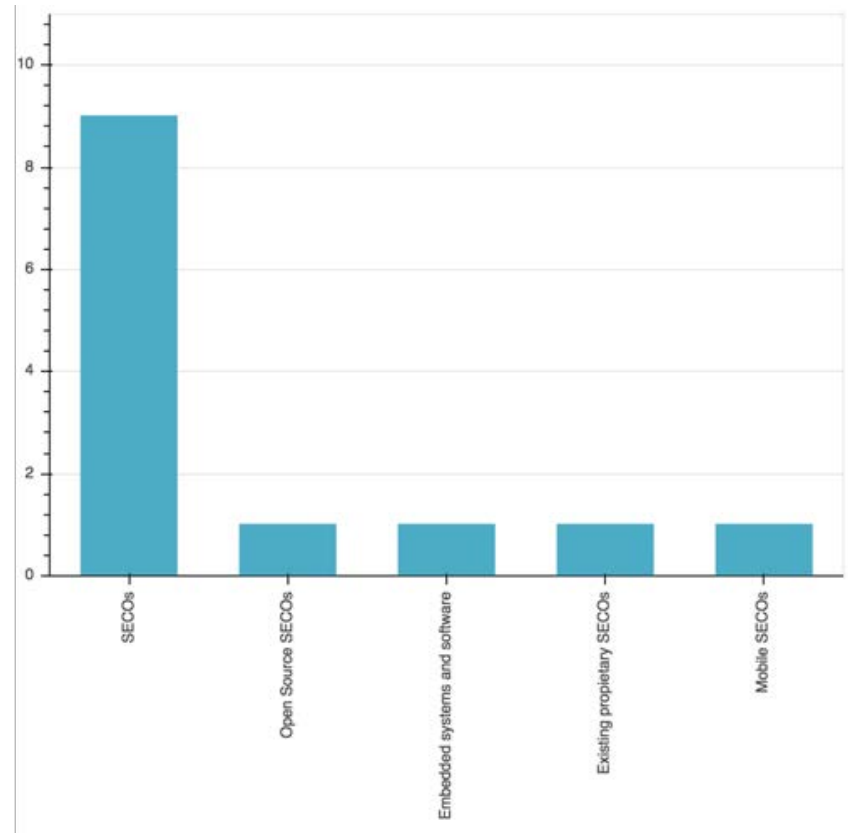

Figure 4. Graph with the number of papers per domain

\subsection{MQ8. Which years cover the review and mapping studies?}

Another point of study in this work is the analysis of the period coyering that the different studies cover. Figure 5 provides an overview of the different periods; the $\mathrm{x}$-axis represents the publication year of the oldest paper, and the $\mathrm{y}$-axis represents the publication year of the newest paper obtained after applying the search protocol in each of the studies.

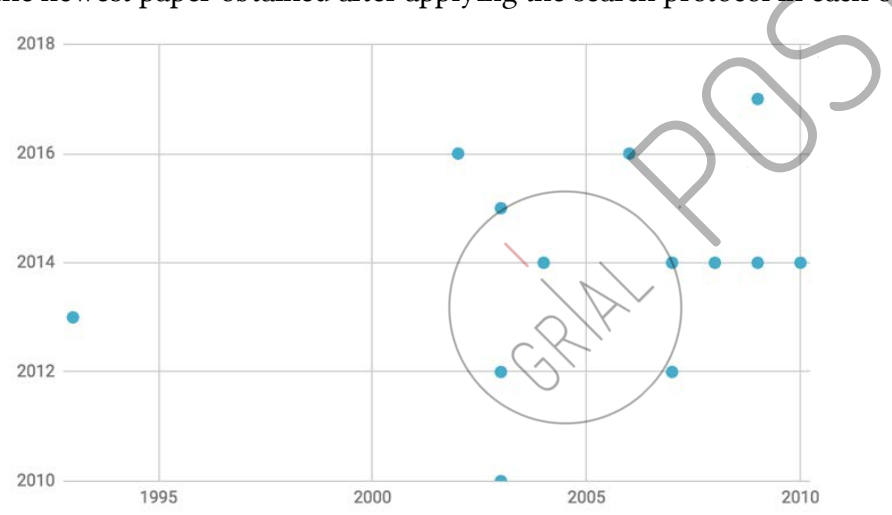

Figure 5. Distribution of time periods covered by each study

\subsection{MQ9. What type of review are published?}

Finally, there are two types of studies, systematic literature review and systematic mapping studies. Also, it is possible to conduct both studies together. Namely, some works combine research questions with mapping questions. There are more mapping studies (7 papers) among the selected papers than systematic reviews (4 papers). Moreover, 2 papers conduct both studies. Table 5 shows the classification of papers according to the type of the study conducted. 
Table 5. Papers classified by type of study

\begin{tabular}{|l|l}
\hline Papers & Type \\
\hline [29] [24] [32] [21] & Review \\
\hline [27] [25] [31] [26] [30] [28] [23] & Mapping \\
\hline [2] [22] & Review and mapping
\end{tabular}

\section{DISCUSSION}

There are 13 selected papers about systematic literature reviews and/or mapping studies about software ecosystems, but each study is focused on different aspects of this kind of solutions (Table 6). For more details, a summary of the main findings of each study is available in [33].

\section{Table 6. Overview of selected studies}

Paper What aspects of the technological ecosystems are analysed?

[23] First systematic mapping about software ecosystems. It identifies the characteristics and a set of limitations and challenges of software ecosystems.

[29] It describes the theoretical foundations of software ecosystems.

[2] Systematic literature review about software ecosystems. It analyses the types of solutions to improve software ecosystems.

[30] It analyses the Key Performance Indicators (KPI). It identifies the goals of software ecosystems in which the KPI are applied.

[32] Systematic review focused on the concept of "ecosystem health", which refers to a set of key indicators of well-being, longevity and performance of the software ecosystem.

[26] It conducts a mapping of the literature focused on different aspects of ecosystems, product lines and strategic innovations in the development of embedded software and systems products.

[31] It provides an overview of mobile software ecosystems (MSECO). They identify their main characteristics and determine the common areas that are investigated in the literature.

[25] Systematic mapping about how software ecosystems are described and documented.

[22] This work is a revisiting of [2].

[21] It performs an analysis of proprietary software ecosystems using the publications identified in the previous SLR.

[27] Systematic mapping study about open source software ecosystems focused on what they are and how they are modelled.

[24] It focuses on a particular aspect of software ecosystems, the governance mechanisms of this type of technological solutions.

[28] It performs a systematic mapping on requirements engineering in software ecosystems. 
Regarding the different domains in which each study is framed, there are two studies provide a general view about the state of research in SECO, a systematic mapping carried out by Barbosa and Alves in 2011 [23], and an SLR conducted by Manikas and Hansen in 2013 [2] and update in 2016 [22]. There is a third work in 2012 about the theoretical foundations of software ecosystems [29], but it is a short paper published in a conference and does not provide a full overview.

Also, there are 4 papers $[21,26,27,31]$ focused on the state of research, but in a particular subdomain of SECO. These papers analyse the characteristics and the state of the art of a specific type of SECO (open source, proprietary, mobile and embedded).

Finally, there are 5 studies that go in depth in a particular characteristic or technique related to software ecosystems: 1 paper about requirements engineering [28]; 1 paper that analyses the governance mechanisms in SECO [24]; 1 paper focused on key performance indicators (KPIs) [30]; 1 paper about "ecosystem health" which refers to a set of key indicators of well-being, longevity and performance of a SECO [32]; and 1 paper about how to document or describe SECOs [25].

The results of this mapping study confirm the statement of Manikas [2], the research in software ecosystems is in its infancy. The distribution of dots in Figure 5 shows the period in which the field of software ecosystems is gaining in importance among the published research. Most of the studies analyse papers published during the last two decades. This is understandable because of the first publication in which appear the software ecosystem concept was in 2005 by Messerschmitt and Szyperski [3]. Moreover, most of the analysed systematic literature reviews and mappings were published during the last four years (Figure 2).

Highlight that all studies are focused on software ecosystems, they put other concepts aside such as digital ecosystems or technological ecosystems. Although some studies include these concepts in their search terms, 11 papers $(84.62 \%$ of the final papers selected) use the software ecosystem term. Furthermore, 4 papers only use software ecosystem term (singular or plural) to build the search string, mainly the studies conducted by Manikas [2, 21, 22].

\section{CONCLUSIONS}

There are several works in the literature that describe a systematic literature review or a mapping study related to software ecosystems. Some studies provide a full overview of this kind of solutions and others delve into specific aspects, but some aspects still need attention. In particular, some studies classify the different approaches to develop software ecosystems, such as architectural patterns or metamodels, but there is no a systematic review focused on this software architectures and model-driven engineering in software ecosystems.

Regarding technological ecosystems, although this concept is framed in the field of software ecosystems, it presents different shades that need solutions focused on the evolution of the ecosystem $[12,13,34]$ and the inclusion of the human factor at the same level of software components [35], but all analysed studies are focused on software ecosystems.

This work provides a base to identify gaps and research opportunities in the area of software ecosystems. It provides a global state of the art through validated studies, taking as reference points the systematic review conducted by Manikas [2, 22] and the systematic mapping by Barbosa and Alves [53].

Finally, the analysis of the different studies lays the groundwork for conducting a systematic literature review focused on solutions in the field of software engineering applied to software ecosystems, with special emphasis on technological ecosystems.

\section{ACKNOWLEDGMENTS}

This research work has been carried ouf within the University of Salamanca Ph.D. Programme on Education in the Knowledge Society scope (http://knowledgesociety.usal.es) and was supported by the Spanish Ministerio de Educación, Cultura y Deporte under an FPU fellowship (FPU014/04783).

This work has been partially funded by the Spanish Government Ministry of Economy and Competitiveness throughout the DEFINES project (Ref. TIN2016-80172-R) and the Ministry of Education of the Junta de Castilla y León (Spain) throughout the T-CUIDA project (Ref. SA061P17).

\section{REFERENCES}

[1] M. Iansiti and R. Levien. 2004. Strategy as ecology. Harvard Business Review 82, 3, 68-78.

[2] K. Manikas and K. M. Hansen. 2013. Software ecosystems - A systematic literature review. Fournal of Systems and Software 86, 5 (05/05/2013), 1294-1306. DOI:http://dx.doi.org/10.1016/j.jss.2012.12.026.

[3] D. G. Messerschmitt and C. Szyperski. 2005. Software ecosystem: understanding an indispensable technology and industry. MIT Press Books 1.

[4] M. Lungu, M. Lanza, T. Gîrba, and R. Robbes. 2010. The Small Project Observatory: Visualizing software ecosystems. Science of Computer Programming 75, 4, $264-275$. DOI:http://dx.doi.org/0.1016/j.scico.2009.09.004. 
[5] M. Lungu. 2008. Towards reverse engineering software ecosystems. In Software Maintenance, 2008. ICSM 2008. IEEE International Conference on IEEE, 428-431.

[6] S. Jansen, A. Finkelstein, and S. Brinkkemper. 2009. A Sense of Community: A Research Agenda for Software Ecosystems. In 31st International Conference on Software Engineering - Companion Volume, 2009. ICSE-Companion 2009. Vancouver, BC, 16-24 May 2009 IEEE, USA, 187-190. DOI:http://dx.doi.org/10.1109/ICSECOMPANION.2009.5070978.

[7] J. Bosch. 2009. From software product lines to software ecosystems. In Proceedings of the 13th International Software Product Line Conference Carnegie Mellon University, San Francisco, California, USA, 111-119.

[8] J. Bosch and P. M. Bosch-Sijtsema. 2010. Softwares product lines, global development and ecosystems: Collaboration in software engineering. In Collaborative Software Engineering Springer, Berlin, Heidelberg, 77-92. DOI:http://dx.doi.org/10.1007/978-3-642-10294-3_4.

[9] K. Pillai, H. King, and C. Ozansoy. 2012. Hierarchy Model to Develop and Simulate Digital Habitat Ecosystem Architecture. In 2012 IEEE Student Conference on Research and Development (SCOReD) IEEE, USA. DOI:http://dx.doi.org/10.1109/SCOReD.2012.6518639.

[10] S. S. Ostadzadeh, F. Shams, and K. Badie. 2015. An architectural model framework to improve digital ecosystems interoperability. In New Trends in Networking, Computing, E-learning, Systems Sciences, and Engineering. Lecture Notes in Electrical Engineering, K. Elleithy and T. Sobh Eds. Springer, Cham, 513-520. DOI:http://dx.doi.org/10.1007/978-3-319-06764-3_65.

[11] J. Shen, L. Zhang, Z. Fan, M. Abbasi, and I. Rafique. 2012. A UML-based software services ecosystem modeling approach. Applied Mechanics and Materials 198-199, 766-771.

[12] A. García-Holgado and F. J. García-Peñalvo. 2013. The evolution of the technological ecosystems: an architectural proposal to enhancing learning processes. In Proceedings of the First International Conference on Technological Ecosystem for Enhancing Multiculturality (TEEM'13) (Salamanca, Spain, November 14-15, 2013) ACM, New York, 565571. DOI:http://dx.doi.org/10.1145/2536536.2536623.

[13] F. J. García-Peñalvo and A. García-Holgado (Ed.). 2017. Open Source Solutions for Knowledge Management and Technological Ecosystems. IGI Global, Hershey.

[14] A. García-Holgado and F. J. García-Peñalvo. 2014. Architectural pattern for the definition of eLearning ecosystems based on Open Source developments. In Proceedings of 2014 International Symposium on Computers in Education (SIIE) (Logroño, La Rioja, Spain, November 12-14, 2014), J.L. Sierra-Rodríguez, J.M. Dodero-Beardo and D. Burgos Eds. Institute of Electrical and Electronics Engineers. IEEE Catalog Number CFP1486T-ART, 93-98. DOI:http://dx.doi.org/10.1109/SIIE.2014.7017711.

[15] B. Kitchenham and S. Charters. 2007. Guidelines for performing Systematic Literature Reviews in Software Engineering. Version 2.3.

[16] K. Petersen, R. Feldt, S. Mujtaba, and M. Mattsson. 2008. Systematic mapping studies in software engineering. In Proceedings of the Proceedings of the 12th international conference on Evaluation and Assessment in Software Engineering (Italy2008). BCS Learning \& Development Ltd., 2227123, 68-77.

[17] K. Petersen, S. Vakkalanka, and L. Kuzniarz. 2015. Guidelines for conducting systematic mapping studies in software engineering: An update. Information and Software Technology 64, 1-18. DOI:http://dx.doi.org/10.1016/j.infsof.2015.03.007.

[18] M. Petticrew and H. Roberts. 2005. Systematic Reviews in the Social Sciences: A Practical Guide. Blackwell Publishing, Malden, USA.

[19] D. Moher, A. Liberati, J. Tetzlaff, D. G. Altman, and Prisma Group. 2009. Preferred reporting items for systematic reviews and meta-analyses: the PRISMA statement. PLoS medicine 6, 7, e1000097. DOI:http://dx.doi.org/10_1371/journal.pmed1000097.

[20] A. García-Holgado and F. J. García-Peñalvo. 2018. Code repository that supports the research presented in the paper "Mapping the systematic literature studies about software ecosystems". DOI:http://dx.doi.org/10.5281/zenodo.1305410.

[21] K. Manikas. 2016. Supporting the Evolution of Research in Software Ecosystems: Reviewing the Empirical Literature. In Software Business. Lecture Notes in Business Information Processing, A. Maglyas and A.L. Lamprecht Eds. Springer, Cham, 63-78.

[22] K. Manikas. 2016. Revisiting software ecosystems Research: A longitudinal literature study. Journal of Systems and Software 117(2016/07/01), 84-103. DOI:http://dx.doi.org/10.1016/j.jss.2016.02.003.

[23] O. Barbosa and C. Alves. 2011. A systematic mapping study on software ecosystems. In 3rd International Workshop on Software Ecosystems 2011, IWSECO 2011 CEUR-WS, Brussels, Belgium, 15-26.

[24] C. Alves, J. Oliveira, and S. Jansen. 2017. Software Ecosystems Governance - A Systematic Literature Review and Research Agenda. In Proceedings of the Proceedings of the 19th International Conference on Enterprise Information Systems (2017). 215-226. DOI:http://dx.doi.org/10.5220/0006269402150226.

[25] O. Pettersson and J. Andersson. 2016. A Survey of Modeling Approaches for Software Ecosystems. In Software Business. Lecture Notes in Business Information Processing, A. Maglyas and A.L. Lamprecht Eds. Springer, Cham, 79-93. 
[26] E. Papatheocharous, J. Andersson, and J. Axelsson. 2015. Ecosystems and Open Innovation for Embedded Systems: A Systematic Mapping Study. In Software Business. ICSOB 2015. Lecture Notes in Business Information Processing, J. Fernandes, R. Machado and K. Wnuk Eds. Springer, Cham, 81-95. DOI:http://dx.doi.org/10.1007/978-3-31919593-3_7.

[27] O. Franco-Bedoya, D. Ameller, D. Costal, and X. Franch. 2017. Open source software ecosystems: A Systematic mapping. Information and Software Technology 91(2017/11/01), 160-185. DOI:http://dx.doi.org/10.1016/j.infsof.2017.07.007.

[28] A. Vegendla, A. N. Duc, S. Gao, and G. Sindre. 2018. A Systematic Mapping Study on Requirements Engineering in Software Ecosystems. Journal of Information Technology Research 11, 1, 49-69. DOI:http://dx.doi.org/10.4018/jitr.2018010104.

[29] G. Hanssen and T. Dybå. 2012. Theoretical foundations of software ecosystems. In Proceedings of the 4th International Workshop on Software Ecosystems 2012, IWSECO 2012 (Cambridge, MA, USA, June 18th, 20122012). CEUR-WS, 6-17.

[30] F. Fotrousi, S. A. Fricker, M. Fiedler, and F. Le-Gall. 2014. KPIs for Software Ecosystems: A Systematic Mapping Study. In Software Business. Towards Continuous Value Delivery. ICSOB 2014. Lecture Notes in Business Information Processing, C. Lassenius and K. Smolander Eds. Springer, Cham, 194-211. DOI:http://dx.doi.org/10.1007/978-3319-08738-2_14.

[31] A. D. L. Fontão, R. P. D. Santos, and A. C. Dias-Neto. 2015. Mobile Software Ecosystem (MSECO): A Systematic Mapping Study. In 2015 IEEE 39th Annual Computer Software and Applications Conference, 653-658. DOI:http://dx.doi.org/10.1109/COMPSAC.2015.121.

[32] S. Hyrynsalmi, M. Seppänen, T. Nokkala, A. Suominen, and A. Järvi. 2015. Wealthy, Healthy and/or Happy - What does 'Ecosystem Health' Stand for? In Software Business. ICSOB 2015. Lecture Notes in Business Information Processing, J. Fernandes, R. Machado and K. Wnuk Eds. Springer, Cham, 272-287. DOI:http://dx.doi.org/10.1007/978-3-319-19593-3_24.

[33] A. García-Holgado. 2018. Análisis de integración de soluciones basadas en software como servicio para la implantación de ecosistemas tecnológicos educativos. In Programa de Doctorado en Formación en la Sociedad del Conocimiento University of Salamanca, Salamanca, Spain.

[34] A. García-Holgado and F. J. García-Peñalvo. 2016. Architectural pattern to improve the definition and implementation of eLearning ecosystems. Science of Computer Programming 129, 20-34. DOI:http://dx.doi.org/10.1016/j.scico.2016.03.010.

[35] A. García-Holgado and F. J. García-Peñalvo. 2018. Human interaction in learning ecosystems based in Open Source solutions. In Learning and Collaboration Technologies. Design, Development and Technological Innovation. 5th International Conference, LCT 2018. Held as Part of HCI International 2018, Las Vegas, NV, USA, Fuly 15-20, 2018 Springer International Publishing, Switzerland. DOI:http://dx.doi.org/10.1007/978-3-319-91743-6_17.

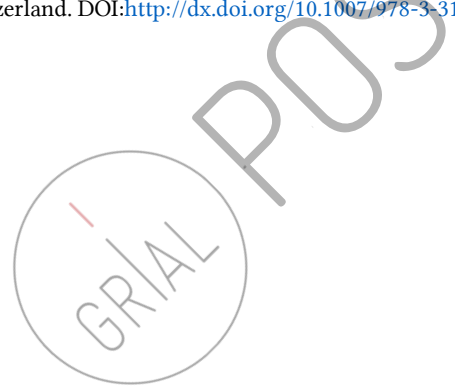

